\title{
A scale-bridging study of the influence of TCP phases on the mechanical properties of an additive manufactured Ni-base superalloy combining microcompression testing, $\mathrm{X}$-ray nanotomography and TEM
}

Michael Sommerschuh ${ }^{1}$, Janis Wirth ${ }^{2}$, Silvan Englisch ${ }^{2}$, Thomas Przybilla ${ }^{2}$, Benjamin Apeleo Zubiri ${ }^{2}$, Julian Pistor ${ }^{3}$, Benoit Merle ${ }^{4}$, Carolin Körner ${ }^{3}$, Mathias Göken ${ }^{4}$ and Erdmann Spiecker ${ }^{2}$

${ }^{1}$ Institute of Micro- and Nanostructure Research (IMN) \& Center for Nanoanalysis and Electron Microscopy (CENEM), Interdisciplinary Center for Nanostructured Films (IZNF), Department of Materials Science and Engineering, Friedrich-Alexander University Erlangen-Nürnberg (FAU), Erlangen, Germany, United States, ${ }^{2}$ Institute of Micro- and Nanostructure Research (IMN) \& Center for Nanoanalysis and Electron Microscopy (CENEM), Interdisciplinary Center for Nanostructured Films (IZNF), Department of Materials Science and Engineering, Friedrich-Alexander University Erlangen-Nürnberg (FAU), Erlangen, Germany, Erlangen, Bayern, Germany, ${ }^{3}$ Chair of Materials Science and Engineering for Metals (WTM), Friedrich-Alexander-University Erlangen-Nürnberg (FAU), Erlangen, Germany, United States, ${ }^{4}$ Materials Science \& Engineering 1, Interdisciplinary Center for Nanostructured Films (IZNF), Friedrich-Alexander-University Erlangen-Nürnberg (FAU), Erlangen, Germany, United States

For the development of single-crystalline Ni-base superalloys with improved properties, it is of high importance to understand the critical parameters governing the morphological impact of topologically close packed (TCP) phases inside the superalloy on the mechanical properties under various loading conditions. Some studies have tackled the tensile properties of conventionally cast alloys at different temperatures and various strain rates or creep properties of such alloys $[1,2]$. There is also research published on (very) high cycle fatigue as well as on TCP-phase quantification in additive manufactured and cast single-crystalline Ni-base superalloys [3,4], but correlative research linking the mechanical properties and the amount of precipitated TCP phases is quite fragmentary [5].

The TCP-phase precipitation strongly depends on the degree of microsegregations of refractory elements, especially $\mathrm{Re}$, in the $\gamma$-phase. By additive manufacturing processes like selective electron beam melting (SEBM), these microsegregations can be significantly reduced, owing to a dramatic increase in the solidification front velocity and the local thermal gradient resulting in a diminution of the primary dendrite arm spacing by more than one order of magnitude. Therefore after employing a short heat treatment to optimize the $\gamma / \gamma^{\prime}$-microstructure, the precipitation of TCP phases is clearly retarded during aging for long times at high temperatures compared to common cast materials [4, 6].

In this work, the morphological and chemical nature of TCP phases and their spatial distribution in single crystalline SEBM material is examined and related to deformation mechanisms leading to mechanical failure in the end. For this purpose, micropillars are extracted from high temperature aged bulk SEBM specimens. To guarantee a sufficiently strong pillar base for the compression experiments, pillars with diameters of about $10 \mu \mathrm{m}$ are milled out of the bulk material using a picosecond 3DMicromac microPREP PRO laser ablation system followed by final focused ion beam (FIB) polishing. Thereby the forces are distributed over a huge supporting structure $(2 \mathrm{~mm} \times 2 \mathrm{~mm})$ composed of the same material, which is glued to a mounting pin for the compression experiments.

The pillars are investigated by combined pillar compression and X-ray nanotomography (Nano-CT) using a Zeiss Xradia Ultra 810 X-ray microscope equipped with an in situ mechanical loading stage 
(Figure 1). The lab-based X-ray microscope with a $\mathrm{Cr}-\mathrm{K} \alpha$-source $(5.4 \mathrm{keV})$ exhibits two imaging modes, the large field-of-view (LFOV) mode with a spatial resolution down to $(150 \mathrm{~nm})^{3}$ in a field of view of $(64 \mu \mathrm{m})^{3}$ and the high-resolution (HRES) mode with a spatial resolution of about $(50 \mathrm{~nm})^{3}$ in a field of view of $(16 \mu \mathrm{m})^{3}$. Moreover, Nano-CT images can be acquired in either absorption or phase contrast mode. Whereas absorption contrast is produced by variations in X-ray attenuation (BeerLambert law) and is therefore well suited for materials exhibiting rather large differences in $\mathrm{Z}$ number, phase contrast is generated by introducing a Zernike phase ring into the beam path behind the sample and Fresnel zone plate. Zernike phase contrast is beneficial for investigation of low- $\mathrm{Z}$ materials or materials with little differences in local density, and further enhances features like edges, pores and precipitates. For Nano-CT imaging of the present superalloy samples, both absorption and Zernike phase contrast can be exploited because TCP phases accumulate a considerable portion of heavy elements like Re from the matrix (Figure 2d), but also deliver a detectable phase shift with respect to the matrix (Figure 1b,c). The HRES mode of the microscope is well suited for the analysis of TCP phases showing typical dimensions not smaller than $100 \mathrm{~nm}$.

Microcompression testing is performed in two different ways. For some of the pillars ex situ microcompression tests are carried using a KLA-Tencor Nanoindenter G200 with a force resolution of $50 \mathrm{nN}$. In order to evaluate the role of the TCP phases during plastic deformation the test is interrupted at different stages of deformation for Nano-CT imaging in the Xradia $810 \mathrm{X}$-ray microscope (Figure 1). Other pillars are compressed in situ in the Zeiss Ultra Load Stage directly mounted in the X-ray microscope (Figure 1d,e). While the former tests are performed under load control the latter are displacement controlled with a minimum step size of $50 \mathrm{~nm}$.

This non-destructive 3D imaging approach enables to study the impact of TCP phases and their 3D distribution (Figure 1c,f) on the mechanical properties of the superalloy in detail providing direct insight into how TCP phases affect, e.g., crystal slip and crack formation (Figure 1f). HRES Nano$\mathrm{CT}$ allows the tomographic reconstruction of nearly a whole primary dendrite arm $(\approx 10-15 \mu \mathrm{m}[4$, $6]$ ) within one pillar $(\approx 10 \mu \mathrm{m}$; Figure 1a) to cover the complete characteristic segregation distance of elements in the additive manufacturing process.

The Nano-CT experiments are complemented by electron diffraction, energy dispersive X-ray spectroscopy (EDXS) and high-resolution (HR) scanning transmission electron microscopy (STEM) experiments conducted in a double aberration-corrected Titan Themis3 60-300 transmission electron microscope equipped with a Super-X EDXS detector. The TCP phases (Figure 2a,c-e) and their impact on the surrounding microstructure are characterized before and after microcompression testing. After completion of the experiment, thin lamellae are site-specifically prepared from the pillars via FIB milling to enable high-resolution TEM analysis of interfaces and defects and their role in local deformation events.

This scale-bridging and complementary approach combining in situ microcompression testing, NanoCT and TEM allows a unique insight into the microstructural behaviour and the influence of TCP phases on the mechanical properties of additive manufactured Ni-base superalloys upon mechanical stress. [7] 


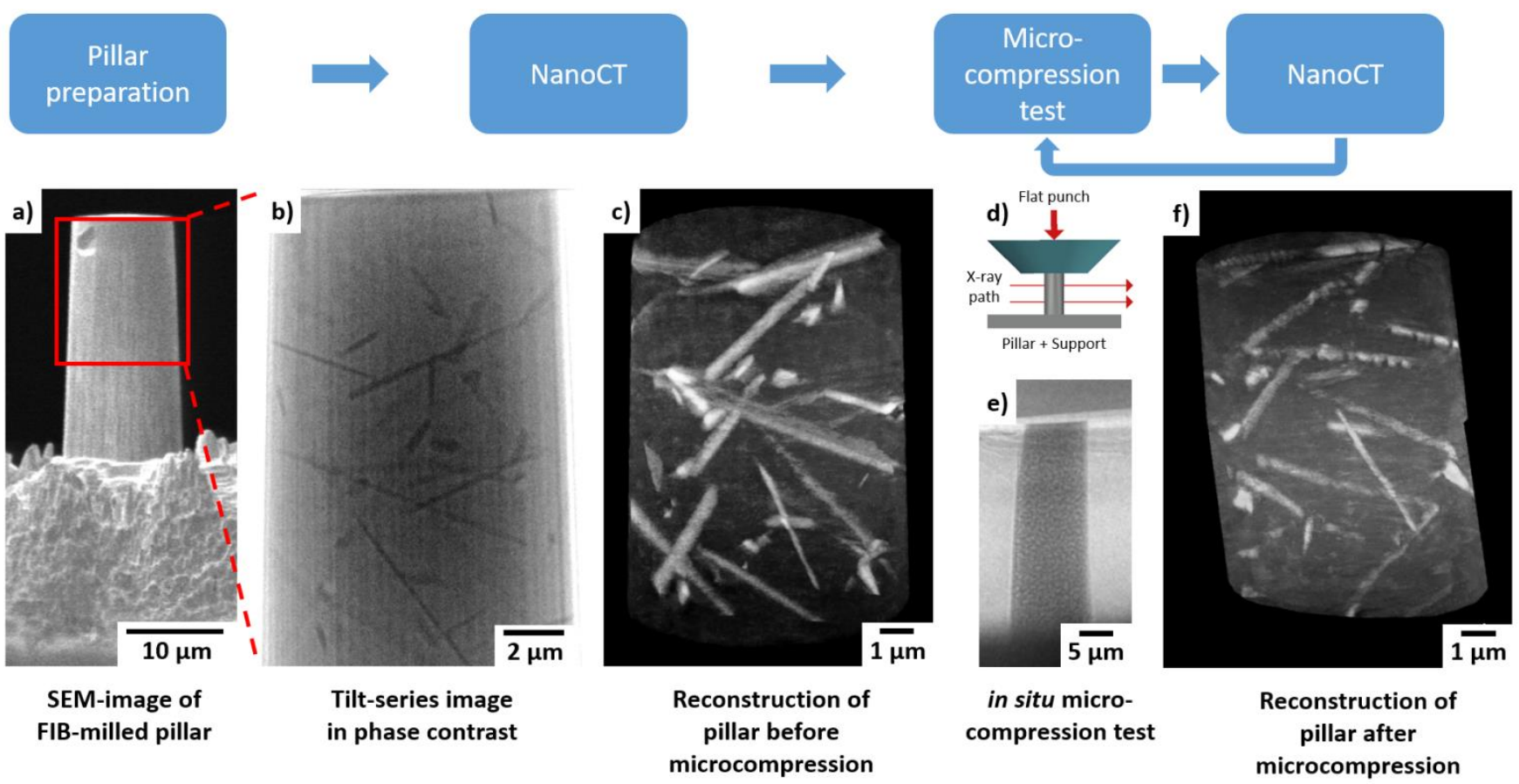

Figure 1. Combined non-destructive Nano-CT and micropillar compression testing of a single crystalline additive manufactured Ni-base superalloy. a) SEM image of a micropillar after FIB-milling, b) Phase contrast image from a HRES Nano-CT tilt series of the marked pillar section (TCP phases appear dark), c) 3D reconstruction of the pillar using SIRT algorithm clearly reveals the TCP phases (bright contrast), d) Schematic drawing of the in situ loading stage setup inside the Zeiss Xradia Ultra 810 X-ray microscope enabling Nano-CT imaging during/after compression, e) Phase contrast LFOV Nano-CT image of a micropillar with the flat compression punch on top, $f$ ) 3D reconstruction of the pillar from $c$ ) after microcompression, clearly revealing fracturing of several TCP phases. 


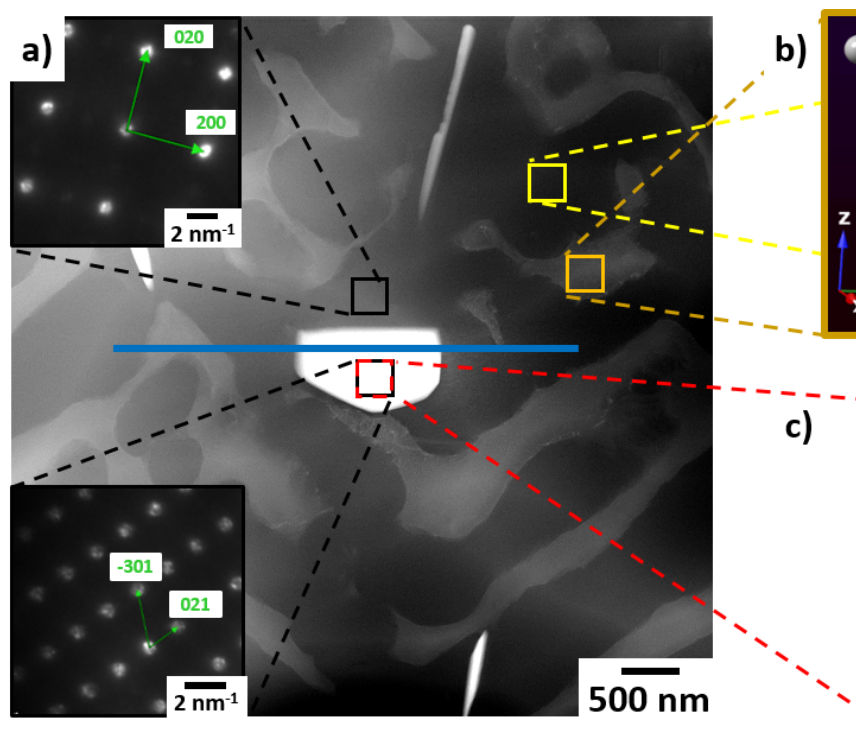

HAADF-STEM image of a typical microstructural section within a pillar

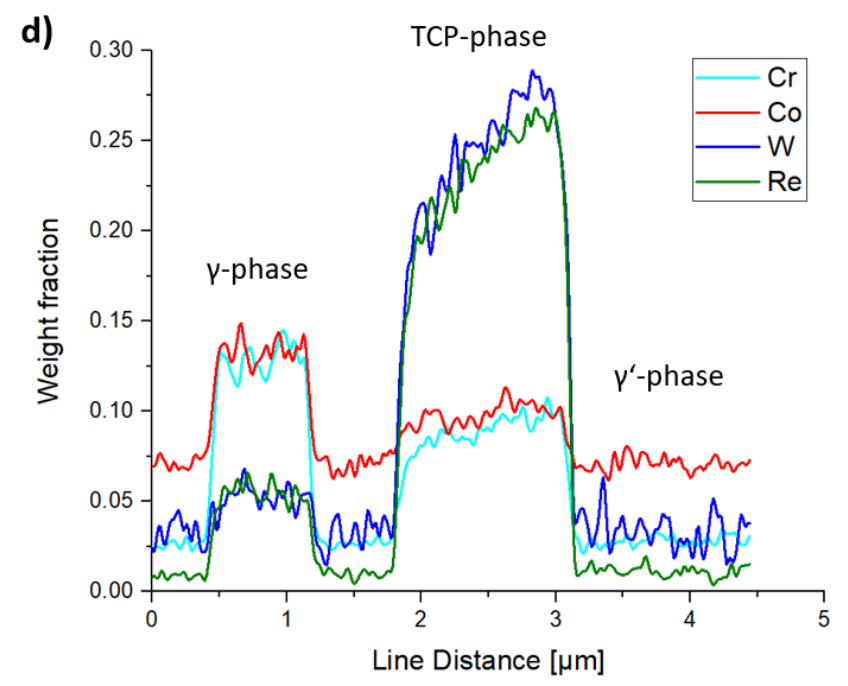

EDX line scan along the blue line in a), accumulated elements in $\boldsymbol{\gamma}$ - and TCP-phase

c)
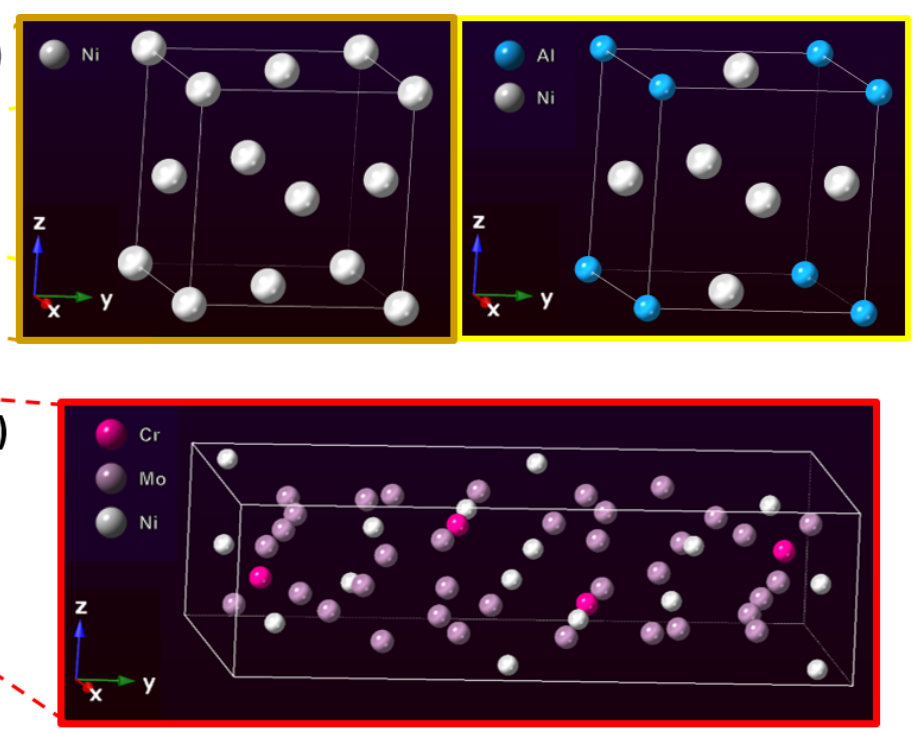

Unit cell of $\gamma$-phase (top left), of $\gamma^{\prime}$-phase (top right), of a TCP-phase (P-phase, bottom)

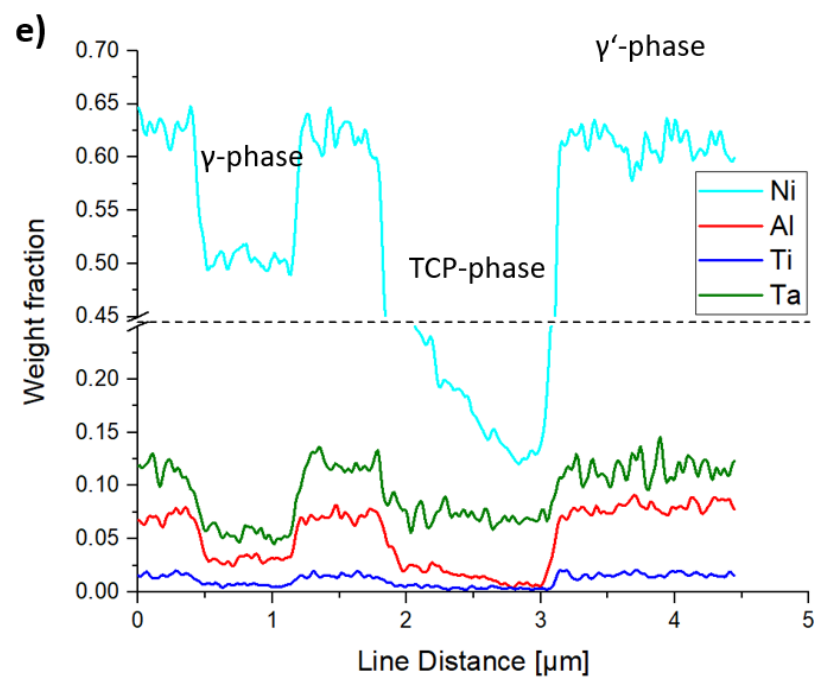

EDX line scan along the blue line in a), depleted elements in $\gamma$ - and TCP-phase

Figure 2. TEM analysis of the microstructure of a single crystalline additive manufactured Ni-base superalloy containing TCP phases. a) High-angle annular dark-field (HAADF) STEM image of the local environment around a TCP phase (bright contrast), the upper inset shows the [001] nanobeam electron diffraction pattern of the $y / \gamma^{\prime}$-matrix, the lower inset shows the [2-36] nanobeam electron diffraction pattern of a TCP phase (P-phase), b) Schematic unit cells of the constituting phases $\gamma, \gamma^{\prime}$ and $c$ ) a TCP phase with the unit cells composed of standard stoichiometric elements, d) and e) EDXS line scans along the blue line in a) unveiling the segregation of elements to the different phases in the microstructure. 


\section{References}

[1] K. Hrutkay, D. Kaoumi, Tensile deformation behavior of a nickel based superalloy at different temperatures, Materials Science and Engineering: A 599 (2014) 196-203.

[2] X.Z. Qin, J. Guo, C. Yuan, G. Yang, L. Zhou, H. Ye, A mu-Phase behavior in a cast Ni-base superalloy, Journal of Materials Science 44 (2009) 4840-4847.

[3] A. Cervellon, S. Hémery, P. Kürnsteiner, B. Gault, P. Kontis, J. Cormier, Crack initiation mechanisms during very high cycle fatigue of Ni-based single crystal superalloys at high temperature, Acta Materialia 188 (2020) 131-144.

[4] J. Pistor, C. Körner, Formation of topologically closed packed phases within CMSX-4 single crystals produced by additive manufacturing, Materials Letters: X 1 (2019) 100003.

[5] P. Caron, Role and behaviour of $\mu$ phase during deformation of a nickel-based single crystal superalloy, Materials Science \& Engineering A 254(1-2) (1998) 1-12.

[6] C. Körner, M. Ramsperger, C. Meid, D. Bürger, P. Wollgramm, M. Bartsch, G. Eggeler, Microstructure and Mechanical Properties of CMSX-4 Single Crystals Prepared by Additive Manufacturing, Metallurgical and Materials Transactions A 49(9) (2018) 3781-3792.

[7] We gratefully acknowledge financial support by the German Research Foundation (DFG) within the frameworks of the research training group GRK1896 "In situ Microscopy with Electrons, X-rays and Scanning Probes", the project SP648/8 "High-resolution X-ray microscopy for correlative tomography, high throughput screening and in situ mechanical testing of structural and functional materials" (project number 316992193) and the projects A6, A7 and B2 of the collaborative research center SFB Transregio/103 "From Atoms to Turbine Blades - a Scientific Approach for Developing the Next Generation of Single Crystal Superalloys" (project number 190389738). 